\title{
A Case Report of Hodgkin's Lymphoma in a Patient with Carcinosarcoma of the Bladder
}

\author{
Anthony Perre Maurie Markman \\ Cancer Treatment Centers of America, Eastern Regional Medical Center, \\ Philadelphia, Pa., USA
}

\section{Key Words}

Hodgkin's lymphoma · Carcinosarcoma - Bladder carcinoma - Pulmonary nodule . Extrathoracic malignancy

\begin{abstract}
The screening of patients with a known primary extrathoracic malignancy for pulmonary metastasis may result in the identification of solitary or multiple pulmonary nodules.

Radiologic features of these pulmonary nodules may suggest a diagnosis, but these features cannot reliably distinguish between benign and malignant etiologies. We present the case of a patient, diagnosed with carcinosarcoma of the bladder, who was found to have multiple pulmonary nodules by $\mathrm{CT}$ evaluation. Physical examination of the patient demonstrated the presence of cervical and axillary lymphadenopathy. An excisional biopsy of an axillary lymph node confirmed the diagnosis of Hodgkin's lymphoma. This case report demonstrates that radiographic information obtained by $\mathrm{CT}$ scan must be carefully correlated with the history and physical examination of the patient. This case report also demonstrates the importance of diagnostic biopsy of pulmonary nodularity discovered in patients with a known primary extrathoracic malignancy. The assumption that these pulmonary nodules represented metastatic malignancies would have had crucial prognostic and therapeutic implications.
\end{abstract}

\section{Introduction}

Improvements in technology have led to increased sensitivity of CT imaging, resulting in the detection of smaller pulmonary nodules. For patients with both an extrathoracic malignancy and indeterminate pulmonary nodules, PET/CT imaging may be useful in predicting the probability of malignancy.

However, in a study by 0 et al. [1] of patients with a known extrathoracic malignancy and indeterminate pulmonary nodules with no FDG uptake, or FDG uptake less than that of the mediastinum, it was found that more than $19 \%$ of the indeterminate 
pulmonary nodules were malignant. Once solitary or multiple pulmonary nodules have been discovered by radiologic testing in patients with a known primary malignancy, there may be radiologic features that suggest a diagnosis, but these features cannot reliably distinguish between benign and malignant etiologies. Features of pulmonary nodules seen in patients with a known primary extrathoracic malignancy associated with malignant rather than benign disease are: (1) the lesion is non-calcified; (2) the lesion is ovoid rather than linear in appearance; (3) the lesion is in close proximity to an adjacent blood vessel; (4) the lesion has reticular changes; (5) the lesion is located at the lung bases and subpleural space, and (6) an increased size of the lesion.

The primary pulmonary venous drainage pattern is characteristic of renal cell, breast, head and neck, and testicular carcinoma. Subsequently, these tumors account for more than $50 \%$ of all lung metastases [2]. Statistically, the most common primary malignancies that result in pulmonary metastasis are from breast, colorectal, and renal cell carcinoma, uterine leiomyosarcoma, and head and neck squamous cell carcinoma [3]. Multiple pulmonary nodules that are large and well circumscribed, 'cannonball metastases', are associated with colorectal carcinoma and sarcoma. Some tumors that have a high number of small metastases, a 'miliary pattern', are associated with thyroid carcinoma, osteosarcoma, and ovarian carcinoma. Both cannon ball and miliary types of pulmonary nodules are associated with renal cell cancer and malignant melanoma.

Metastatic disease may present as a single metastasis. This is most frequently seen in colorectal carcinoma. It may also be seen in osteosarcoma, carcinoma of the kidney, testicle or breast, and malignant melanoma [4]. Metastases that tend to hemorrhage (e.g. choriocarcinoma, angiosarcoma, renal cell carcinoma, melanoma, thyroid carcinoma, and Kaposi's sarcoma) may have indistinct borders. Cavitation occurs in less than $5 \%$ of malignant lesions, and is most commonly seen in squamous cell carcinoma from a head and neck, or lung origin, sarcoma, and after treatment. Further evaluation depends on clinical presentation as well as physical examination of the patient. The American College of Chest Physicians recommends that, in patients who are candidates for curative treatment, each nodule be evaluated individually, as necessary, and that curative treatment not be denied unless there is histopathologic confirmation of metastasis [5].

We present the case of a patient, diagnosed with a primary bladder carcinoma, who was found to have Hodgkin's lymphoma presenting as multiple pulmonary nodules. After treatment with ABVD, there were favorable anatomic and metabolic responses to therapy, as evidenced by resolution of previously hypermetabolic cervical lymphadenopathy, and significant improvement in axillary, intrathoracic and intraabdominal disease.

\section{Case Presentation}

Our patient is a 57-year-old man with a past medical history significant for chronic left anterior and posterior cervical lymphadenopathy, which had begun 20 years earlier. Biopsy of these lymph nodes had been performed many years ago and, by patient report, had demonstrated benign disease. The cervical lymphadenopathy had been stable in size for many years.

The patient was in his usual state of health until June 2011 when he experienced a sudden onset of gross hematuria. He went to the emergency room, and a CT scan of the abdomen and pelvis was 
performed. The CT scan demonstrated multiple pulmonary nodules located in the left lung base, in addition to several bilateral sub-centimeter pulmonary nodules. There was non-specific lymphadenopathy in the retroperitoneum as well as a $1.9-\mathrm{cm}$ mass-like density seen adjacent to the left posterior bladder, suggesting malignancy. A CT scan of the chest was performed and demonstrated multiple pulmonary nodules bilaterally, suggesting metastatic disease. The largest nodule was $3.1 \mathrm{~cm}$ and was located in the AP window. Other nodules measured $1.6,2.0$, and $0.8 \mathrm{~cm}$.

The patient then underwent a cystoscopy with transurethral resection of the bladder tumor. Numerous clots were evacuated. The bladder was inspected several times. There was a slightly elevated 1-cm mass lateral to the left urethral orifice. This lesion was resected at its base and fulgurated. Pathology was consistent with carcinosarcoma. The tumor had invaded the lamina propria. The muscularis propria was not present on evaluation. Microscopic examination demonstrated the presence of multiple malignant spindle-shaped cells with inflammatory changes. Immunohistochemical stains were performed. CK-5/6 and CK-9 were strongly positive. P-63 and thrombomodulin were focally positive. CK-7, CK-20, and uroplakin were negative. These slides were also reviewed at an outside university pathology department, and the pathologic diagnosis was confirmed.

On June 9, 2011, the patient underwent a CT-guided biopsy of a left lower lobe pulmonary nodule. Pathology of the nodule was non-diagnostic. The patient presented to our clinic for a second opinion regarding treatment as he had been advised to undergo treatment for metastatic bladder carcinoma. On examination, left cervical lymphadenopathy was palpated. The cervical lymph nodes were $1 \mathrm{~cm}$ in size. Axillary lymphadenopathy was also palpated.

On June 27, 2011, a PET/CT scan demonstrated multiple hypermetabolic lymph nodes in the neck. A left axillary lymph node was hypermetabolic with an SUV of 18. There were multiple hypermetabolic lesions noted in the paratracheal, prevascular and hilar lymph nodes bilaterally. A right lower lobe mass, $3.8 \mathrm{~cm}$, was hypermetabolic with an SUV of 14.2. There were small nodules in the left cardiophrenic angle and left diaphragmatic pleura that were without associated metabolic activity. Additional hypermetabolic lymphadenopathy was identified in the abdomen.

On June 29, 2011, a core biopsy of a left-sided axillary lymph node was non-diagnostic. An excisional biopsy of a left axillary node was performed on July 7, 2011, and demonstrated classical Hodgkin's lymphoma. The cells were CD30 positive. Bone marrow aspiration and a biopsy performed on July 15, 2011, demonstrated involvement of marrow with Hodgkin's lymphoma. Cystoscopy and repeat biopsies of the bladder, performed on the same day as the bone marrow aspiration, revealed no abnormalities.

On July 25, 2011, the patient started chemotherapy (ABVD). After 4 cycles of ABVD, a PET/CT scan on October 3,2011 , demonstrated favorable anatomic and metabolic responses to the therapy, as evidenced by resolution of previously hypermetabolic cervical lymphadenopathy, and significant improvement in axillary, intrathoracic and intra-abdominal disease.

\section{Discussion}

Radiologic features of pulmonary nodules may suggest a diagnosis, but these features are frequently unreliable. In patients with a known extrathoracic malignancy and multiple small pulmonary nodules, the reported rate of malignancy in these nodules, based on several studies, has been widely variable.

Patz et al. [6] retrospectively reviewed all percutaneous lung biopsy specimens over a 6-year period. A total of 146 patients with a single known primary malignancy and multiple pulmonary nodules were biopsied. Of these biopsy specimens, $93.7 \%$ were positive for metastatic disease. The authors concluded that, in patients who have a known primary malignancy and multiple pulmonary nodules, biopsy of these nodules will rarely change the clinical course. They also concluded that the overwhelming 
majority of patients studied could have been treated based on the pre-biopsy presumptive diagnosis.

Other studies have indicated that the likelihood of pulmonary nodules representing malignancy in patients with a known extrathoracic malignancy is not as high as compared to what was reported in the study by Patz et al. [6]. In a study by Ginsberg et al. [7], VATS was performed in patients with pulmonary nodules measuring less than 1 $\mathrm{cm}$. When a single pulmonary nodule was resected via VATS in patients who had a known malignancy, $59 \%$ of those nodules were found to be malignant. When multiple pulmonary nodules were resected via VATS in patients who had a known malignancy, $64 \%$ of patients had at least one nodule which was found to be malignant [7].

The size of the pulmonary nodules in patients with a known malignancy may be predictive in determining the likelihood of malignancy. In the study by Ginsberg et al. [7], in patients with a known primary malignancy, $84 \%$ of pulmonary nodules with a diameter more than $1 \mathrm{~cm}$ were malignant, as opposed to $49 \%$ of pulmonary nodules with a diameter less than $1 \mathrm{~cm}$. Although increased size of pulmonary nodules may be predictive of malignancy, $42 \%$ of pulmonary nodules smaller than $0.5 \mathrm{~cm}$ were still found to be malignant.

A study by Khokhar et al. [8] evaluated the medical records of 151 patients who had a history of non-thoracic malignancy and the presence of non-calcified pulmonary nodules. Out of those 151 patients, 64 (42\%) were diagnosed with malignant nodules, 32 had newly diagnosed lung cancers, 28 had metastatic spread of their primary cancers, and 4 had either cancers that were new or of undetermined etiology. On multivariable analysis, nodule size and previous tobacco exposure were predictive of malignancy.

Quint et al. [9] evaluated the charts of patients with a known extrathoracic malignancy and who were found to have a solitary pulmonary nodule by chest imaging. Patients with carcinoma of the head and neck, breast, cervix, bile ducts, esophagus, prostate or stomach were more likely to have primary bronchogenic carcinoma rather than lung metastasis. Patients with carcinoma of the salivary glands, adrenal glands, colon, parotid gland, kidney, thyroid, thymus, and uterus had fairly even odds. Patients with melanoma, sarcoma, or testicular carcinoma were more likely to have a solitary metastasis than a bronchogenic carcinoma. This study suggests that the primary malignancy type may be predictive of the pulmonary nodule's etiology. These findings suggest the need for clinical correlation, and a low threshold for diagnostic biopsy in patients with extrapulmonary cancers and non-calcified pulmonary nodules.

In this particular case, the specific pattern or features of the pulmonary nodules viewed in historical and clinical contexts may have been helpful in determining diagnosis.

Non-Hodgkin's lymphoma may present as multiple pulmonary nodules. Pulmonary nodules from non-Hodgkin's lymphoma are preferentially found in the lower lobes, and are occasionally surrounded by a halo of ground-glass attenuation, or contain air bronchograms. Enlarged mediastinal or hilar lymph nodes may not be present. NonHodgkin's lymphoma may also be seen as plaque-like or nodular densities in the pleura. Pleural effusion is seen in $25 \%$ of patients with lymphoma [10]. 
Hodgkin's lymphoma involving the lung may present in a variety of radiographic patterns, including well-defined or ill-defined pulmonary nodules. Among patients with Hodgkin's lymphoma, 67-74\% have abnormal radiographic findings at presentation. In those patients with abnormal radiographic findings, $90 \%$ have bilateral asymmetrical disease. Prevascular and paratracheal lymph nodes are most commonly affected in Hodgkin's lymphoma.

As demonstrated in this case study, radiographic information obtained by CT scan should be considered in the context of the patient's history and physical examination. For our patient, the results of the diagnostic biopsy had critical prognostic and therapeutic implications. Prognostically, a patient who has carcinosarcoma of the bladder metastatic to the lung has a far different life expectancy as compared to a patient with two separate and potentially curable malignancies. In addition, chemotherapy used in the setting of metastatic carcinosarcoma of the bladder would have been substantially different from the treatment used in this patient to ultimately attain clinical remission.

As the sensitivity of radiologic studies continues to improve, clinicians are more often likely to be confronted with the diagnostic dilemma of pulmonary nodularity discovered in patients with a known primary extrathoracic malignancy. This case illustrates that although radiologic features of pulmonary nodularity may help predict probability of malignancy, ultimately these characteristics are unreliable, and that it is critically important to perform a diagnostic biopsy of these nodules in patients with a known primary extrathoracic malignancy.

\section{References}

$10 \mathrm{JH}$, et al: Clinical significance of small pulmonary nodules with little or no 18F-FDG uptake on PET/CT images of patients with nonthoracic malignancies. J Nucl Med 2007;48:15-21.

2 Gilbert HA, Kagan AR: Metastasis: incidence, detection, and evaluation without histologic confirmation; in Weiss L (ed): Fundamental Aspects of Metastasis. Amsterdam, North-Holland, 1976, pp 385-405.

- 3 Seo JB, Im JG, Goo JM, et al: Atypical pulmonary metastasis: spectrum of radiologic findings. Radiographics 2001;21:403-417.

4 Collins J, Stern EJ: Chest Radiology: The Essentials. Philadelphia, Lippincott, Williams and Wilkins, 2007.

5 Gould M, et al: Evaluation of patients with pulmonary nodules: when is it lung cancer? ACCP evidencebased clinical practice guidelines (2nd edition). Chest 2007;132(3 suppl):108S-130S.

6 Patz EF, et al: Significance of percutaneous needle biopsy in patients with multiple pulmonary nodules and a single known primary malignancy. Chest 1995;107:601-604.

7 Ginsberg MS, et al: Pulmonary nodules resected at video-assisted thorascopic surgery: etiology in 426 patients. Radiology 1999;213:277-282.

8 Khokhar S, et al: Significance of non-calcified pulmonary nodules in patients with extrapulmonary cancers. Thorax 2006;61:331-336.

9 Quint LE, et al: Solitary pulmonary nodules in patients with extrapulmonary neoplasms. Radiology 2000;217:257-261

10 Dunnick NR, Reaman GH, Head GL, et al: Radiographic manifestations of Burkitt's lymphoma in American patients. AJR Am J Roentgenol 1979;132:1-6. 